\section{Automated diffraction tomography in TEM and STEM mode - getting the best of both worlds}

\author{
Ute Kolb ${ }^{1}$, Sergi Plana-Ruiz ${ }^{2}$, Yasar Krysiak ${ }^{1}$, Haishuang Zhao ${ }^{1}$ \\ 1. Johannes Gutenberg University Mainz, Mainz, Germany \\ 2. Universitat de Barcelona, Barcelona, Spain \\ email: kolb@uni-mainz.de
}

In the last decade electron crystallography experienced a boost through the development of electron diffraction tomography. After data acquisition and processing strategies were developed for automated diffraction tomography [1] a big range of methods has been set up in order to improve the collection of data in reciprocal space. A major difference between the methods is the crystal tracking strategy carried out in TEM or STEM mode. While the use of TEM for electron diffraction tomography methods allows the application of both, selected area or nano-beam diffraction, the STEM mode will only facilitate the later. Both data collection approaches already proved successful for structure solution and refinement using single nano- sized crystals. Significant differences appear for example if the size of crystalline domains are in the regime of some tens of nanometer or the material is highly beam sensitive. The use of a small condenser aperture $(10 \mu \mathrm{m})$ to produce a quasi-parallel electron probe allows the access of these materials as a nanometer-beam $(2-10 \mathrm{~nm})$ with low convergence angle (0.4-1 mrad) reduces the electron dose using dark-field detectors for crystal imaging in scanning mode [2]. Automated diffraction tomography utilizes in addition the advantage that only a slight change of focus allows to switch between imaging and diffraction conditions [2]. In order to obtain improved electron diffraction data electron beam precession using this beam configuration is added. This allows electron diffraction data acquisition with expanded number of excited spots and with further minimized dynamical effects suitable for crystal structure refinement using kinematical and dynamical approach. For the above described data collection strategy it is necessary to obtain an accurate alignment of such a beam setting, which may not be available as a default implemented option in a TEM. Thus a general method to set illuminating conditions for selectable beam convergence and probe size has been works out for Transmission Electron Microscopes (TEM) fitted with $\mu \mathrm{s} /$ pixel fast beam scanning control, (S)TEM, and an annular dark field detector [3]. Additionally, scripts for different TEM platforms addressing the above described data collection strategies were developed.

References:

[1] U. Kolb, T. Gorelik, M.T. Otten, (2008) Ultramicroscopy, 108, 763-772

[2] U. Kolb, T. Gorelik, C. Kübel, M.T. Otten and D. Hubert, (2007) Ultramicroscopy, 107, 507-513

[3] S.Plana-Ruiz, Portillo, S.Estradé, F.Peiró, UteKolb,

S.Nicolopoulos, (2018) Ultramicroscopy, 193, 39-51

Keywords: electron diffraction tomography, scanning transmission electron microscopy, structure solution
MS24 Defects and disorder quantification at the nanoscale

Chairs: Dr. Partha Pratim Das, Dr. Tatiana Gorelik

MS24-01

\section{Defects, disorder and electrochemistry in layered metal-ion battery cathodes}

\section{Artem Abakumov}

1. Center for Electrochemical Energy Storage, Skolkovo Institute of Science and Technology, Moscow, Russia

email: a.abakumov@skoltech.ru

Layered rock-salt-type oxides $\mathrm{A}_{1+\mathrm{x}} \mathrm{M}_{1-\mathrm{x}} \mathrm{O}_{2}$ (A - alkali cation, $\mathrm{M}$ - transition metal cation) are among the most promising positive electrode (cathode) materials for metal-ion batteries, offering specific capacity up to $250 \mathrm{mAh} / \mathrm{g}$. The parent structure of the $\mathrm{A}_{1+\mathrm{x}} \mathrm{M}_{1-\mathrm{x}} \mathrm{O}_{2}$ oxides is based on "cubic" close packing of oxygen atoms where the $\mathrm{A}$ and $\mathrm{M}$ cations occupy octahedral interstices in an ordered manner forming alternating layers. On a local scale, however, the structure of these materials is much more complex, featuring different kinds of defects and partial disorder, related to fractional occupation of the octahedral positon in the M layers with the excessive A cations $(\mathrm{x}>0)$ with subsequent $\mathrm{A} / \mathrm{M}$ ordering, antisite $\mathrm{A} / \mathrm{M}$ disorder, stacking faults in the $\mathrm{A} / \mathrm{M}$ ordered layers and twinning of the close packed layers. Electrochemical extraction of the A cations introduces intricate evolution of the defect structure, related to gliding of the close packed layers with respect to each other, partially reversible migration of the $\mathrm{M}$ cations to the vacant positions in the A-layers with subsequent ordering to a spinel-type structure, trapping of the $\mathrm{M}$ cations in the tetrahedral interstices, anion vacancies due to oxygen loss at high potentials and surface structure "densification" due to the oxygen loss or even a conversion to a framework structures. The defect structure of the pristine, charged, discharged and cycled $\mathrm{A}_{1+\mathrm{x}} \mathrm{M}_{1-\mathrm{x}} \mathrm{O}_{2}$ materials will be demonstrated using aberration-corrected scanning transmission electron microscopy and correlated with the peculiarities of their electrochemical behavior, such as operating potential, capacity and voltage fade $[1-3]$.

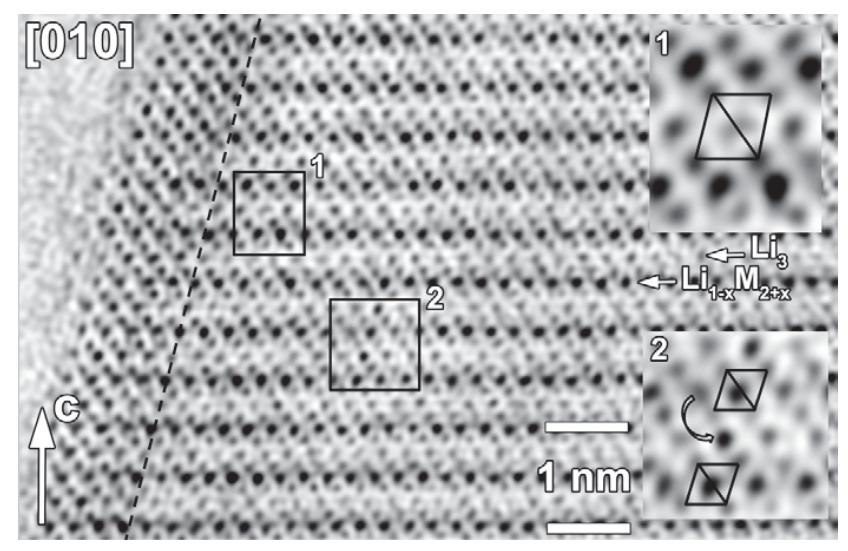

